\title{
Une nouvelle définition de l'entropie dynamique des systèmes non commutatifs
}

\author{
Jean-Luc Sauvageot et Jean-Paul Thouvenot \\ Laboratoire de Probabilités, Université Pierre et Marie Curie, Tour 56, 4 place Jussieu, \\ F-75252 Paris Cedex 05, France
}

Reçu le 1 octobre 1991

\begin{abstract}
An alternative definition of entropy for non-commutative systems, equivalent to the one by A. Connes, H. Narnhofer, and W. Thirring, is given. It is based on the concepts of conditional entropy, and stationary couplings with classical systems. It allows to prove that any quantum dynamical system with singular spectrum has zero entropy.
\end{abstract}

\section{Introduction}

Un système dynamique non commutatif $(\mathbb{A}, \theta, \varrho)$ consiste en une algèbre d'opérateurs $\mathbb{A}$ (ici, une $C^{*}$-algèbre), un automorphisme $\theta$ de $\mathbb{A}$ et un état $\varrho$ qui est $\theta$-invariant sur $\mathbb{A}$.

L'entropie d'un tel système a été définie par Connes et Stormer [4] dans le cas où l'état $\varrho$ est une trace, en suivant la démarche du cas classique (commutatif). Le cas où $\varrho$ n'est pas tracial s'avère plus complexe; la définition de l'entropie dynamique demande une procédure originale (Connes, [2] et Connes, Narnhofer et Thirring [3]), que l'on peut reformuler en termes de «mesure physique»:

La mesure des phénomène quantiques s'opère en couplant un système quantique $(\mathbb{A}, \varrho)$ avec un appareil de mesure classique, c'est à dire un espace topologique probabilisé $(X, \mu)$. Le couplage se formalise comme un état $\lambda$ sur l'algèbre produit tensoriel $\mathbb{A} \otimes \mathbb{B}[\operatorname{avec} \mathbb{B}=C(X)$, l'algèbre des fonctions continues sur $X]$, de marges respectives $\varrho$ et $\mu$ sur $\mathbb{A}$ et $\mathbb{B}$; le couplage $\lambda$ apportera d'autant plus d'information, à travers $(\mathbb{B}, \mu)$, sur l'état du système $\mathbb{A}$ qu'il sera plus éloigné de l'état tensoriel $\varrho \otimes \mu$.

Un tel couplage étant fixé, à toute famille $\left(\mathbb{A}_{0}, \ldots, \mathbb{A}_{n}\right)$ de «sous-algèbres» de dimension finie de $\mathbb{A}^{(1)}$ et toute famille $\left(\mathbb{P}_{0}, \ldots, \mathbb{P}_{n}\right)$ de sous-algèbres de dimension

\footnotetext{
1 Par «sous-algèbre de dimension finie,» il faut entendre plus précisément une algèbre de dimension finie et une application complètement positive $\gamma$ de celle-ci dans $\mathbb{A}$, préservant l'unité. Toutes les algèbres sont supposées posséder une unité, quitte à en rajouter une
} 
finie de $\mathbb{B}$ (autrement dit, de partitions de $X$ ), est associée une information mutuelle $\varepsilon_{\lambda}\left(\mathbb{A}_{0}, \ldots, \mathbb{A}_{n} ; \mathbb{P}_{0}, \ldots, \mathbb{P}_{n}\right)$ qui mesure la quantité d'information passée des $\mathbb{A}_{i}$ vers les $\mathbb{P}_{i}$ via l'appareillage $\lambda$.

L'entropie conjointe $H_{\varrho}\left(\mathbb{A}_{0}, \ldots, \mathbb{A}_{n}\right)$ est définie comme le supremum de ces informations mutuelles; l'entropie dynamique $h_{\varrho}\left(\mathbb{A}_{0}, \theta\right)$ d'un sous-système fini est la limite des $\frac{1}{n+1} H_{\varrho}\left(\mathbb{A}_{0}, \ldots, \mathbb{A}_{n}\right)$ lorsque les $\mathbb{A}_{i}=\theta^{i}\left(\mathbb{A}_{0}\right)$ sont les itérées par $\theta$ de $\mathbb{A}_{0}$; enfin, l'entropie dynamique $h_{\varrho}(\theta)$ du système $(\mathbb{A}, \theta, \varrho)$ est le suprémum des ces $h_{\rho}\left(\mathbb{A}_{0}, \theta\right)$.

Cet article propose une simplification de la procédure, dont le principe consiste à limiter la classe des couplages autorisés.

On ne considèra que des couplages stationnaires:

- d'une part, l'appareil de mesure $(X, \mu)$ est lui-même un système dynamique classique, soit $X=P^{\mathbb{Z}}$, la mesure $\mu$ étant invariante par le shift $\sigma$ de $X$;

- d'autre part, le couplage $\lambda$ est un état sur $\mathbb{A} \otimes C(X)$ qui est invariant par l'automorphisme $\theta \otimes \sigma$.

On obtient ainsi une nouvelle définition de l'entropie dynamique, ne faisant plus référence aux sous-systèmes de dimension finie dans la $C^{*}$-algèbre $\mathbb{A}$. En notant $\mathbb{P}$ la sous-algèbre de $\mathbb{B}$ (ou la partition de $X$ ) correspondant à la coordonnée $z=0$ de $P^{\mathbb{Z}}$, au couplage stationnaire $\lambda$ est associé ce qu'on pourrait appeler une information dynamique mutuelle:

$$
h(\mathbb{P}, \lambda)=H_{\mu}\left(\mathbb{P} \mid \mathbb{P}^{-}\right)-H_{\lambda}\left(\mathbb{P} \mid \mathbb{P}^{-} \otimes \mathbb{A}\right)
$$

$\left[\mathbb{P}^{-}\right.$est le passé de $\mathbb{P}$ et $H_{\mu}\left(\mathbb{P} \mid \mathbb{P}^{-}\right)$est l'entropie dynamique de la partition $\mathbb{P}$ dans le système classique; l'entropie conditionnelle $H_{\lambda}\left(\mathbb{P} \mid \mathbb{P}^{-} \otimes \mathbb{A}\right)$ est définie par référence à l'entropie relative de Araki $[1] . h(\mathbb{P}, \lambda)$ s'interprète comme la "quantité d'information dynamique» passée du système quantique $(\mathbb{A}, \theta, \varrho)$ dans le système classique $(X, \sigma, \mu)$ à travers $\lambda]$.

L'entropie du système dynamique $(\mathbb{A}, \theta, \varrho)$ sera le suprémum des $h(\mathbb{P}, \lambda)$ sur tous les couplages stationnaires possibles.

Le premier avantage de cette approche est qu'elle s'affranchit de l'hypothèse de nucléarité présente dans [3]. La difficulté en revanche consiste à démontrer, dans le cas nucléaire, que cette définition a priori plus restrictive coïncide avec la définition originale de Connes, Narnhofer et Thirring (cf. Proposition 4.1 cidessous).

Le second avantage est de rendre aisée la démonstration d'une propriété fondamentale de l'entropie dynamique, celle d'être liée au «spectre de Lebesgue»: on montre donc que, lorsque l'unitaire implémentant $\theta$ a un spectre singulier, l'entropie est nécessairement nulle (Proposition 5.1). Un résultat dans ce sens a déjà été obtenu par Stormer et Voiculescu [6]. Il ne concerne que les automorphismes de Bogolioubov sur l'algèbre des relations d'anticommutation, mais il dit nettement plus dans la mesure où il suffit que le spectre soit singulier sur la base pour que l'entropie soit nulle, alors que le type spectral maximal est l'exponentielle de convolution de cette mesure.

\section{Entropie relative}

1.1. Rappel de la définition (cf. Araki, [1]): Mest une algèbre de von Neumann, $\varrho$ un état fidèle de $\mathbb{M}$; sa représentation G.N.S. est notée $\left(\mathscr{H}_{\varrho}, \pi_{\varrho}, \xi_{\varrho}\right)$. 
Soit $\omega$ une forme linéaire positive normale sur $\mathbb{M}$ : la forme quadratique qui, à l'élément $\pi_{e}(x) \xi_{e}$ de $\mathscr{H}_{Q}(x \in \mathbb{M})$, associe le nombre $\omega\left(x x^{*}\right)$, est fermable, et sa fermeture détermine un opérateur positif auto-adjoint $\Delta_{\omega, \varrho}$ dans $\mathscr{H}_{\varrho}$, appelé Hamiltonien relatif.

L'entropie relative $S(\varrho, \omega)$ est définie par la formule:

$$
S(\varrho, \omega)=\left\langle\xi_{\varrho}, \Delta_{\omega, \varrho} \log \left(\Delta_{\omega, \varrho}\right) \xi_{\ell}\right\rangle .
$$

Lorsque $\mathbb{A}$ est une $C^{*}$-algèbre, $\varrho$ un état de $\mathbb{A}, \omega$ une forme linéaire positive sur $\mathbb{A}$, on définit $S(\varrho, \omega)$ de la manière suivante:

- si le support de $\omega$ est inférieur au support de $\varrho$ dans l'algèbre de von Neumann enveloppante $\mathbb{A}^{* *}$ (ce qui est le seul cas que nous rencontrerons), on prolonge $\varrho$ et $\omega$ à l'algèbre de von Neumann $\mathbb{M}$ réduite de $\pi_{\varrho}(\mathbb{A})^{\prime \prime}$ sur le support de $\varrho$, où l'on applique la définition précédente;

- sinon, on pose $S(\varrho, \omega)=-\infty$.

\subsection{Propriétés}

1.2.1. On a

$$
\begin{aligned}
& S(\varrho, \varrho)=0, \\
& S(\varrho, \omega) \in[0,+\infty] \text { si } \omega \text { est un état }([1], 1.3), \\
& S(\varrho, \alpha \omega)=\alpha S(\varrho, \omega)+\alpha \log \alpha \cdot \omega\left(1_{\mathbb{M}}\right), \quad \forall \alpha \geqq 0, \omega \in \mathbb{M}_{*}^{+} .
\end{aligned}
$$

Lorsque $\omega$ est fidèle, on déduit de [1], 2.1 et 3.1, la formule:

$$
S(\varrho, \omega)=i \frac{d}{d t} \omega\left[(D \varrho: D \omega)_{t}\right]_{\mid t=0}
$$

dont on déduit sans trop de difficulté le lemme suivant:

1.3. Lemme. Soit $\left\{\omega_{1}, \ldots, \omega_{n}\right\}$ une famille de formes linéaires positives normales sur $\mathbb{M},\left\{\alpha_{1}, \ldots, \alpha_{n}\right\}$ une famille de nombres positifs de somme 1 :

a) on a $\sum_{i} S\left(\varrho, \omega_{i}\right)=S\left(\varrho, \Sigma_{i} \omega_{i}\right)+\sum_{i} S\left(\Sigma_{j} \omega_{j}, \omega_{i}\right)$;

b) si les $\left\{\omega_{i}\right\}$ sont des états, on a, en posant $\omega=\sum \alpha_{i} \omega_{i}$ :

$$
\sum \alpha_{i} S\left(\varrho, \omega_{i}\right)=S(\varrho, \omega)+\sum \alpha_{i} S\left(\omega, \omega_{1}\right) .
$$

(On retrouve en particulier l'inégalité de convexité:

$$
\left.S\left(\varrho, \sum \alpha_{i} \omega_{i}\right) \leqq \sum \alpha_{i} S\left(\varrho, \omega_{i}\right) .\right)
$$

Démonstration. On ne démontre que a), dont b) se déduit par 1.2.1 ci-dessus.

Cas particulier. On suppose les $\omega_{i}$ fidèles, et on écrit, en posant $\omega=\sum \omega_{i}$ :

$$
\begin{aligned}
\sum \frac{d}{d t} \omega_{i}\left[\left(D \varrho: D \omega_{i}\right)_{t}\right]_{\mid t=0} & =\sum \frac{d}{d t} \omega_{i}\left[(D \varrho: D \omega)_{t}\left(D \omega: D \omega_{i}\right)_{t}\right]_{\mid t=0} \\
& =\sum \frac{d}{d t} \omega_{i}\left[(D \varrho: D \omega)_{t}\right]_{\mid t=0}+\sum \frac{d}{d t} \omega_{i}\left[\left(D \omega: D \omega_{i}\right)_{t}\right]_{\mid t=0} \\
& =\frac{d}{d t} \omega\left[(D \varrho: D \omega)_{t}\right]_{\mid t=0}+\sum \frac{d}{d t} \omega_{i}\left[\left(D \omega: D \omega_{i}\right)_{t}\right]_{\mid t=0}
\end{aligned}
$$


D'où le résultat par 2.1.2.

Cas général. On applique le cas particulier aux $\omega_{i}^{\prime}=\omega_{i}+\varepsilon \varrho$, et on fait tendre $\varepsilon$ vers 0 .

\section{Couplages et entropie conditionnelle}

2.1. Définitions. On se donne une $C^{*}$-algèbre $\mathbb{A}$ et un état $\varrho$ de $\mathbb{A}$.

On appelle couplage de $(\mathbb{A}, \varrho)$ avec une $C^{*}$-algèbre abélienne $\mathbb{B}$ un état $\lambda$ sur la $C^{*}$-algèbre produit tensoriel $\mathbb{A} \otimes \mathbb{B}$, de marge $\varrho$ sur $\mathbb{A}$.

Un couplage $\lambda$ induit une mesure $\mu$ sur le spectre $X$ de $\mathbb{B}$.

Lorsque $\mathbb{B}$ est de dimension finie, à chaque point $x$ de $X$ sont associés

- un projecteur minimal $\chi_{x}$ de $\mathbb{B}$,

- une forme linéaire positive $\omega_{x}\left\{a \rightarrow \lambda\left(a \otimes \chi_{x}\right)\right\}$ sur $\mathbb{A}$ et un état $\varrho_{x}=\frac{1}{\mu\{x\}} \cdot \omega_{x}$.

On obtient ainsi une décomposition

$$
\varrho=\sum_{x \in X} \omega_{x}=\sum_{x \in X} \mu\{x\} \varrho_{x}
$$

de l'état initial $\varrho$.

L'information mutuelle associée à un couplage $\lambda$ de $\mathbb{A}$ avec une algèbre abélienne $\mathbb{B}$ de dimension finie (cf. [3], II.4) est le nombre

$$
\varepsilon_{\lambda}(\mathbb{A}, \mathbb{B})=\sum_{x \in X} \mu\{x\} S\left(\varrho, \varrho_{x}\right) ;
$$

et l'entropie conditionnelle est définie comme:

$$
H_{\lambda}(\mathbb{B} \mid \mathbb{A})=H_{\mu}(\mathbb{B})-\varepsilon_{\lambda}(\mathbb{A}, \mathbb{B})=-\sum_{x \in X} S\left(\varrho, \omega_{x}\right) .
$$

On a $0 \leqq \varepsilon_{\lambda}(\mathbb{A}, \mathbb{B}) \leqq H_{\mu}(\mathbb{B})$ et $0 \leqq H_{\lambda}(\mathbb{B} \mid \mathbb{A}) \leqq H_{\mu}(\mathbb{B})$.

$\left[H_{\mu}(\mathbb{B})=H(\mu)\right.$ est l'entropie classique de l'espace fini $X=$ spectre de $\mathbb{B}$, sous la mesure $\mu]$.

La quantité $\varepsilon_{\lambda}(\mathbb{A}, \mathbb{B})$ est croissante en ses deux arguments.

L'entropie conditionnelle $H_{\lambda}(\mathbb{B} \mid \mathbb{A})$ (appelée entropy defect dans [3]) est croissante et sous-additive en $\mathbb{B}$, décroissante en $\mathbb{A}$.

Heuristiquement, il faut comprendre l'information mutuelle comme un substitut de la quantité $H_{e}(\mathbb{A})+H_{\mu}(\mathbb{B})-H_{\lambda}(\mathbb{A} \otimes \mathbb{B})$ (c'est à dire de quantités du type $H(P)$ $+H(Q)-H(P \vee Q)$ dans le cas classique). Elle peut également s'écrire

$$
\varepsilon_{\lambda}(\mathbb{A}, \mathbb{B})=S(\varrho \otimes \mu, \lambda),
$$

c'est à dire la distance, mesurée en termes d'entropie relative, qui sépare $\lambda$ d'un état tensoriel.

Par partition (finie) de la $C^{*}$-algèbre abélienne $\mathbb{B}$ avec unité, on entendra indifféremment une partition (finie) $P$ du spectre de $\mathbb{B}$, ou encore une sous-algèbre $\mathbb{P}$ (de dimension finie) de $\mathbb{B}$, contenant l'unité, ou enfin une famille (finie) de projecteurs de $\mathbb{B}$, de somme $1_{\mathbb{B}}$.

Si $\lambda$ est un couplage de $\mathbb{A}$ avec $\mathbb{B}$, il réalise également un couplage de $\mathbb{A} \otimes \mathbb{B}$ avec $\mathbb{B}$, via le morphisme canonique de $\mathbb{B} \otimes \mathbb{B}$ dans $\mathbb{B}$ qui, à une fonction de deux variables, associe sa restriction à la diagonale; ainsi, si $\mathbb{P}$ et $\mathbb{Q}$ sont deux partitions finies de $\mathbb{B}$, on obtient par restriction un couplage de $\mathbb{A}$ avec $\mathbb{P}$, avec $\mathbb{Q}$, et avec la 
partition $\mathbb{P} \vee \mathbb{Q}$ engendrée par $\mathbb{P}$ et $\mathbb{Q}$, mais aussi un couplage de $\mathbb{A} \otimes \mathbb{P}$ avec $\mathbb{Q}$; ce qui donne un sens au lemme suivant:

2.2. Lemme. Si $\lambda$ est un couplage de $\mathbb{A}$ avec $\mathbb{B}$, et si $\mathbb{P}$ et $\mathbb{Q}$ sont deux partitions finies dans $\mathbb{B}$, on a

$$
H_{\lambda}(\mathbb{P} \vee \mathbb{Q} \mid \mathbb{A})=H_{\lambda}(\mathbb{P} \mid \mathbb{A})+H_{\lambda}(\mathbb{Q} \mid \mathbb{A} \otimes \mathbb{P}) .
$$

Démonstration. Conséquence immédiate du Lemme 1.3 et des définitions.

2.3. Lemme. $S i\left(\lambda_{i}\right)_{i=1, \ldots, m}$ est une famille de couplages de $\mathbb{A}$ avec une $C^{*}$-algèbre abélienne $\mathbb{B}$ de dimension finie, et si $\left(\alpha_{i}\right)_{i=1, \ldots, m}$ est une famille de nombres positifs de somme 1, on a

$$
\varepsilon_{\Sigma \alpha_{i} \lambda_{i}}(\mathbb{A}, \mathbb{B}) \leqq \sum \alpha_{i} \varepsilon_{\lambda_{i}}(\mathbb{A}, \mathbb{B}) .
$$

Démonstration. Soit $\mathbb{D}$ une $C^{*}$-algèbre abélienne de dimension $m$, de projecteurs minimaux $\left\{p_{1}, \ldots, p_{m}\right\}$, et $\lambda$ le couplage de $\mathbb{A}$ avec $\mathbb{B} \otimes \mathbb{D}$ défini par

$$
\lambda\left(a \otimes b \otimes p_{i}\right)=\alpha_{i} \lambda_{i}(a \otimes b), \quad a \in \mathbb{A}, b \in \mathbb{B}, 1 \leqq i \leqq m .
$$

On vérifie alors

$$
\sum \alpha_{i} \varepsilon_{\lambda_{i}}(\mathbb{A}, \mathbb{B})=\varepsilon_{\lambda}(\mathbb{A} \otimes \mathbb{D}, \mathbb{B}) \geqq \varepsilon_{\lambda}(\mathbb{A}, \mathbb{B})=\varepsilon_{\sum \alpha_{i} \lambda_{i}}(\mathbb{A}, \mathbb{B})
$$

\section{Couplages stationnaires et entropie dynamique}

3.1. Définitions. Soit $(\mathbb{A}, \theta, \varrho)$ un système dynamique non commutatif: $\mathbb{A}$ est une $C^{*}$-algèbre, $\theta$ un automorphisme de $\mathbb{A}$ et $\varrho$ un état $\theta$-invariant sur $\mathbb{A}$.

Un couplage stationnaire du système dynamique $(\mathbb{A}, \theta, \varrho)$ avec un système dynamique classique $(\mathbb{B}, \sigma)$ est un état sur la $C^{*}$-algèbre $\mathbb{A} \otimes \mathbb{B}$ qui est $\theta \otimes \sigma$ invariant, et dont la restriction à $\mathbb{A}$ est égale à $\varrho$.

Si $\mathbb{P}$ est une partition finie dans $(\mathbb{B}, \sigma)$, on notera $\mathbb{P}^{-}$le passé de $\mathbb{P}$, soit $\mathbb{P}^{-}=\bigvee_{i \geqq 1} \sigma^{-i} \mathbb{P}$.

A un couplage stationnaire $\lambda$ avec $(\mathbb{B}, \sigma)$, et une partition finie $\mathbb{P}$ de $\mathbb{B}$, sont associés les nombres

$$
h(\mathbb{P}, \lambda)=H_{\mu}\left(\mathbb{P} \mid \mathbb{P}^{-}\right)-H_{\lambda}\left(\mathbb{P} \mid \mathbb{A} \otimes \mathbb{P}^{-}\right)
$$

et

$$
h^{\prime}(\mathbb{P}, \lambda)=H_{\mu}\left(\mathbb{P} \mid \mathbb{P}^{-}\right)-H_{\lambda}(\mathbb{P} \mid \mathbb{A})
$$

(où $\mu$ est la restriction de $\lambda$ à $\mathbb{B}$, donc une mesure $\sigma$-invariante).

On a $h(\mathbb{P}, \lambda) \geqq 0$ et $h(\mathbb{P}, \lambda) \geqq h^{\prime}(\mathbb{P}, \lambda)$.

L'entropie du système dynamique $(\mathbb{A}, \theta, \varrho)$ est définie comme

$$
h_{\varrho}(\theta)=\sup h(\mathbb{P}, \lambda),
$$

le suprémum étant pris sur tous les couplages stationnaires de $\mathbb{A}$ avec les systèmes dynamiques classiques, puis, pour chaque $\lambda$, sur toutes les partitions finies dans le système clasique.

3.2. Lemme. On a $h_{\varrho}(\theta)=\sup ^{\prime}(\mathbb{P}, \lambda)$. le suprémum étant pris de la même manière.

Démonstration. L'inégalité $h_{\varrho}(\theta) \geqq \sup h^{\prime}(\mathbb{P}, \lambda)$ est immédiate [on a toujours $h(\mathbb{P}, \lambda)$ $\left.\geqq h^{\prime}(\mathbb{P}, \lambda)\right]$. 
On fixe $\varepsilon>0$ et un couplage $\lambda$ de $\mathbb{A}$ avec $(\mathbb{B}, \sigma)$, où $\mathbb{B}$ est l'algèbre des fonctions continues sur $P^{\mathbb{Z}}, P$ étant un espace fini, et $\sigma$ le shift, de sorte qu'en notant $\mathbb{P}_{k}$ la partition de $\mathbb{B}$ correspondant à la coordonnée $k(k \in \mathbb{Z})$, on ait $h\left(\mathbb{P}_{0}, \lambda\right)>h_{\boldsymbol{Q}}(\theta)-\varepsilon$.

On a $H\left(\mathbb{P} \mid \mathbb{P}^{-}\right)=\lim _{n} \frac{1}{n} H\left(\bigvee_{1}^{n} \mathbb{P}_{k}\right)$ et $H\left(\mathbb{P} \mid \mathbb{A} \otimes \mathbb{P}^{-}\right)=\lim _{n} \frac{1}{n} H\left(\bigvee_{1}^{n} \mathbb{P}_{k} \mid \mathbb{A}\right)(\operatorname{par} 2.2) ;$ on fixe $m$ tel que, pour tout $n \geqq m$, les deux limites soient approchées à $\varepsilon$ près, et $\frac{1}{m} \log (m)+\frac{m-1}{m} \log \left(\frac{m-1}{m}\right)<\varepsilon$.

Soit $Y$ l'espace $\mathbb{Z} / m \mathbb{Z}$ muni de la probabilité de comptage $v$, et $\tau$ la translation $z \rightarrow z+1(\bmod m)$. On couple $\mathbb{A}$ avec $\mathbb{B} \otimes C(Y)$ par $\lambda \otimes v$, qui est un état $\theta \otimes \sigma \otimes \tau-$ invariant.

On donne un nom: $\mathbb{Q}=\left\{Q_{\alpha}\right\}_{\alpha \in I}$ aux atomes de la partition $\mathbb{Q}=\bigvee_{1}^{m} \mathbb{P}_{k}$; et on construit une partition $\mathbb{Q}=\left\{\widetilde{Q}_{\alpha}\right\}_{\alpha \in I \cup\{\infty\}}$ de $P^{\mathbb{Z}} \times Y$ en posant:

$$
\tilde{Q}_{\alpha}=Q_{\alpha} \times\{0\} \quad \text { si } \quad \alpha \in I \text {; } \text { et } \tilde{Q}_{\infty}=P^{\mathbb{Z}} \times\{1, \ldots, m-1\} \text {. }
$$

La partition $\bigvee_{0}^{m p-1}(\sigma \otimes \tau)^{k} \mathbb{Q}$ a pour trace $\bigvee_{i+1}^{m p+i} \sigma^{k} \mathbb{P}$ sur l'atome $\{i\}$ de $Y$ $(i=0, \ldots, m-1)$, et a donc pour entropie $H\left(\bigvee_{0}^{m p-1^{i+1}} \sigma^{k} \mathbb{P}\right)+\log (m)$. En divisant par $m p$ et en faisant tendre $p$ vers l'infini, on trouve

$$
H_{\mu \otimes v}\left(\widetilde{\mathbb{Q}} \mid \widetilde{\mathbb{Q}}^{-}\right)=H_{\mu}\left(\mathbb{P} \mid \mathbb{P}^{-}\right) \text {. }
$$

D'autre part, si $\varrho=\sum_{\alpha \in I} \mu_{\alpha} \varrho_{\alpha}$ est la décomposition convexe de l'état $\varrho$ induite par le couplage avec $\mathbb{Q}=\bigvee_{1}^{m} \mathbb{P}_{k}$, comme en 2.1 .1 ci-dessus, la décomposition relative au couplage avec $\widetilde{Q}$ s'écrit:

ce qui donne

$$
\varrho=\frac{1}{m} \sum_{\alpha \in I} \mu_{\alpha} \varrho_{\alpha}+\frac{m-1}{m} \varrho,
$$

$$
H(\widetilde{Q} \mid \mathbb{A})=\frac{1}{m} H(\mathbb{Q} \mid \mathbb{A})+\frac{1}{m} \log (m)+\frac{m-1}{m} \log \left(\frac{m-1}{m}\right)
$$

et $H(\widetilde{Q} \mid \mathbb{A}) \leqq H\left(\mathbb{P} \mid \mathbb{P}^{-} \otimes \mathbb{A}\right)+2 \varepsilon$.

D’où $h^{\prime}(\widehat{\mathbb{Q}}, \lambda \otimes v) \geqq h(\mathbb{P}, \lambda)-2 \varepsilon \geqq h_{\varrho}(\theta)-3 \varepsilon$.

3.3. Proposition. Pour tout entier $m$, on a $h_{\varrho}\left(\theta^{m}\right)=|m| \cdot h_{\varrho}(\theta)$.

Démonstration. Le résultat est immédiat pour $m=0$ et $m=-1$; on peut donc supposer $m>1$.

On considère un couplage stationnaire $\lambda \operatorname{de}(\mathbb{A}, \theta, \varrho)$ avec $(\mathbb{B}, \sigma)$, et la partition $\mathbb{P}$ de $\mathbb{B}$; on pose $\mathbb{Q}=\bigvee_{0}^{m-1} \sigma^{k} \mathbb{P}$. On calcule

$$
\begin{aligned}
h(\mathbb{P}, \lambda) & =\lim _{n} \frac{1}{n} \varepsilon_{\lambda}\left(\bigvee_{1}^{n} \sigma^{k} \mathbb{P}, \mathbb{A}\right)=\lim _{n} \frac{1}{n m} \varepsilon_{\lambda}\left(\bigvee_{1}^{n m} \sigma^{k} \mathbb{P}, \mathbb{A}\right) \\
& =\frac{1}{m} \lim _{n} \frac{1}{n} \varepsilon_{\lambda}\left(\bigvee_{1}^{n} \sigma^{m k} \mathbb{Q}, \mathbb{A}\right) \leqq \frac{1}{m} \cdot h_{\varrho}\left(\theta^{m}\right) .
\end{aligned}
$$

D'où l'inégalité $h_{\varrho}\left(\theta^{m}\right) \geqq m \cdot h_{\varrho}(\theta)$. 
Réciproquement, on fixe $\varepsilon>0$, un couplage stationnaire $\lambda$ du système dynamique $\left(\mathbb{A}, \theta^{m}, \varrho\right)$ avec $(\mathbb{B}, \sigma)$, et $\mathbb{P}=\left\{P_{\alpha}\right\}_{\alpha \in I}$ une partition finie de $\mathbb{B}$ tels que

$$
h_{\lambda}(\mathbb{P}, \lambda) \geqq h_{\ell}\left(\theta^{m}\right)-\varepsilon \text {. }
$$

On pose $Y=\{0, \ldots, m-1\} ; X$ désigne le spectre de $\mathbb{B}$. Sur $X \times Y$, on considère l'automorphisme $\tilde{\sigma}$ défini par

$$
\tilde{\sigma}(x, i)=(x, i-1) \quad \text { si } \quad x \in X, i>0 \text {; et } \quad \tilde{\sigma}(x, 0)=(\sigma x, m-1) .
$$

On considère ensuite le couplage stationnaire $\tilde{\lambda} \operatorname{de}(\mathbb{A}, \theta, \varrho)$ avec $(\mathbb{B} \otimes C(Y), \tilde{\sigma})$ défini par

$$
\tilde{\lambda}(a \otimes b \otimes\{i\})=\lambda\left(\theta^{i} a \otimes b\right), \quad a \in \mathbb{A}, b \in \mathbb{B}, 0 \leqq i \leqq m-1 ;
$$

puis la partition $\mathbb{Q}=\left\{Q_{\alpha}\right\}_{\alpha \in I} \cup Q_{\infty}$ de $X \times Y$, définie par

$$
Q_{\alpha}=P_{\alpha} \times\{m-1\}, \alpha \in I \text {, et } X \times\{0, \ldots, m-2\} \text {. }
$$

On a alors, pour tout $n, \bigvee_{0}^{m n-1} \tilde{\sigma}^{k} \mathbb{Q}=\left(\bigvee_{0}^{n-1} \sigma^{k} \mathbb{P}\right) \otimes Y(Y$ désigne ici la partition en points de $Y$ ); d'où on déduit

$$
\frac{1}{n m} \varepsilon_{\tilde{\lambda}}\left(\bigvee_{0}^{n m-1} \sigma^{k} \mathbb{Q}, \mathbb{A}\right)=\frac{1}{n m} \varepsilon_{\lambda}\left(\bigvee_{0}^{n-1} \sigma^{k} \mathbb{P}, \mathbb{A}\right)
$$

et, en faisant tendre $n$ vers l'infini

$$
h_{\varrho}(\theta) \geqq \frac{1}{m} \cdot\left(h_{\varrho}\left(\theta^{m}\right)-\varepsilon\right) .
$$

\section{Comparaison avec l'entropie de Connes-Narnhofer-Thirring}

L'entropie de [3] est définie de la manière suivante:

On se donne $n$ couples $\left(\mathbb{A}_{i}, \gamma_{i}\right)$, où $\mathbb{A}_{i}$ est une $C^{*}$-algèbre de dimension finie, et $\gamma_{i}$ une application complètement positive de $\mathbb{A}_{i}$ dans $\mathbb{A}$ préservant l'unité. $A$ tout couplage $\lambda$ de $\mathbb{A}$ avec une $C^{*}$-algèbre abélienne $\mathbb{B}$, et toute famille $\left(\mathbb{P}_{1}, \ldots, \mathbb{P}_{n}\right)$ de partitions finies de $\mathbb{B}$, est associée une information mutuelle:

$$
\varepsilon_{\lambda}\left(\gamma_{1}, \ldots, \gamma_{n} ; \mathbb{P}_{1}, \ldots, \mathbb{P}_{n}\right)=H_{\mu}\left(\bigvee_{k=1}^{n} \mathbb{P}_{k}\right)-\sum H_{\lambda_{k}}\left(\mathbb{P}_{k} \mid \mathbb{A}_{k}\right)
$$

où $\mu$ est la trace de $\lambda$ sur $\mathbb{B}$, et $\lambda_{k}$ le couplage induit de $\mathbb{A}_{k}$ avec $\mathbb{B}$.

L'entropie absolue $H_{\varrho}\left(\gamma_{1}, \ldots, \gamma_{n}\right)$ est le suprémum de ces informations mutuelles pour tous les couplages et tous les choix possibles de $\left(\mathbb{P}_{k}\right)$.

A toute application complètement positive unitale $\gamma$ d'une $C^{*}$-algèbre de dimension finie dans $\mathbb{A}$ est associée l'entropie dynamique

$$
h_{\boldsymbol{Q}}(\gamma, \theta)=\lim _{n \rightarrow \infty} \frac{1}{n+1} H_{\mathrm{Q}}\left(\gamma, \theta \circ \gamma, \ldots, \theta^{n} \circ \gamma\right) .
$$

Enfin, l'entropie dynamique de l'automorphisme $\theta$ est définie comme le suprémum des $h_{\boldsymbol{e}}(\gamma, \theta)$ pour tous les $\gamma$ possibles.

4.1. Proposition. Si la $C^{*}$-algèbre $\mathbb{A}$ est nucléaire, l'entropie définie au paragraphe précédent coïncide avec l'entropie de Connes-Narnhofer-Thirring (cf. [3, Def. V.1]). 
Démonstration. Notons $\eta$ l'entropie $h_{\varrho}(\theta)$ définie au par. 3.1 ci dessus, et $\kappa$ l'entropie $h_{\varrho}(\theta)$ définie en [3, V.1].

On remarquera d'abord que, pour un couplage stationnaire $\lambda$ de $\mathbb{A}$ avec $\mathbb{B}$ et une partition finie $\mathbb{P}$ dans $\mathbb{B}$, on a, en posant $\mathbb{P}_{i}=\sigma^{i} \mathbb{P}$ :

$$
\varepsilon_{\lambda}\left(\gamma, \theta \circ \gamma, \ldots, \theta^{n} \circ \gamma ; \mathbb{P}_{0}, \ldots, \mathbb{P}_{n}\right)=H_{\mu}\left(\bigvee_{0}^{n} \mathbb{P}_{k}\right)-(n+1) H\left(\mathbb{P} \mid \mathbb{A}_{0}\right),
$$

soit, en divisant par $(n+1)$ et en passant à la limite:

$$
H_{\mu}\left(\mathbb{P} \mid \mathbb{P}^{-}\right)-H_{\lambda}\left(\mathbb{P} \mid \mathbb{A}_{0}\right) \leqq h_{\varrho}(\gamma, \theta) \leqq \kappa .
$$

Ceci étant vrai pour tout morphisme $\gamma: \mathbb{A}_{0} \rightarrow \mathbb{A}$, on aura

$$
h^{\prime}(\mathbb{P}, \lambda)=H_{\mu}\left(\mathbb{P} \mid \mathbb{P}^{-}\right)-H_{\lambda}(\mathbb{P} \mid \mathbb{A}) \leqq \kappa
$$

et le Lemme 3.2 nous donne l'inégalité $\eta \leqq \kappa$.

Reste à démontrer l'inégalité inverse.

Pour cela, on fixe $\varepsilon>0$, une $C^{*}$-algèbre $\mathbb{A}_{0}$ de dimension finie et une application complètement positive unitale $\gamma$ de $\mathbb{A}_{0}$ dans $\mathbb{A}$ vérifiant

$$
\lim _{n \rightarrow \infty} \frac{1}{n+1} H_{\varrho}\left(\gamma, \theta \circ \gamma, \ldots, \theta^{n} \circ \gamma\right) \geqq \kappa-\varepsilon .
$$

1ère étape (cf. [3, Lemme VI.I]):

On note $X$ l'espace (compact) des états de $\mathbb{A}$.

On fixe un nombre $\delta>0$ vérifiant:

$$
\forall \omega, \omega^{\prime} \in A, \quad\left\|\omega-\omega^{\prime}\right\| \leqq \delta \Rightarrow\left|S(\varrho \circ \gamma, \omega \circ \gamma)-S\left(\varrho \circ \gamma, \omega^{\prime} \circ \gamma\right)\right| \leqq \varepsilon .
$$

On fixe une fois pour toutes une partition finie $\mathbb{R}=\left\{R_{1}, \ldots, R_{d}\right\}$ de $X$, à $d$ éléments, suffisamment fine pour que l'on ait:

si $\omega$ et $\omega^{\prime}$ sont deux états de $\mathbb{A}$ qui appartiennent au même atome de $\mathbb{R}$, alors $\left\|\omega-\omega^{\prime}\right\| \leqq \delta$.

Soit $v$ une mesure de probabilités sur $X$, de barycentre $\varrho$ : $\mathbb{A}$ est naturellement couplée à $C(X)$ par l'état $\Lambda_{v}: a \otimes f \rightarrow \int_{X} \omega(a) f(\omega) d v(\omega)$.

Pour $\Lambda=\Lambda_{v}$, on a $\varepsilon_{\Lambda}\left(\mathbb{A}_{0}, \mathbb{R}\right)=\sum_{1}^{d} v\left(R_{i}\right) S\left(\varrho \circ \gamma, \varrho_{i} \circ \gamma\right)$, où $\varrho_{i}$ est le barycentre de la mesure de probabilité induite par $v$ sur l'atome $R_{i}$ de $\mathbb{R}$. On remarquera que, par le choix de $\delta$ et $\mathbb{R}$, le nombre $S\left(\varrho \circ \gamma, \varrho_{i} \circ \gamma\right)$ est, à $\varepsilon$ près, indépendant du choix de $v$, et que par conséquent la quantité $\sum_{.1}^{d} v\left(R_{i}\right) S\left(\varrho \circ \gamma, \varrho_{i} \circ \gamma\right)$ est, à $\varepsilon$ près, affine en $v$ :

Si on se donne une famille $v_{1}, \ldots, v_{n}$ de mesures sur $X$ de barycentre $\varrho$, une famille $\alpha_{1}, \ldots, \alpha_{n}$ de nombres positifs de somme 1 , alors, en posant $v=\sum \alpha_{i} v_{i}$, on obtient

$$
\left|\varepsilon_{\Lambda_{v}}\left(\mathbb{A}_{0}, \mathbb{R}\right)-\sum \alpha_{k} \varepsilon_{\Lambda_{\nu_{k}}}\left(\mathbb{A}_{0}, \mathbb{R}\right)\right| \leqq \varepsilon .
$$

On posera $\mathbb{X}=C\left(X^{\mathbb{Z}}\right)$, et on notera $\mathbb{R}_{k}$ la partition de $X^{\mathbb{Z}}$ (et donc de $\mathbb{X}$ ) image réciproque de $\theta^{k}(\mathbb{R})$ par la coordonnée d'ordre $k$.

A tout couplage $\lambda$ de $\mathbb{A}$ avec une $C^{*}$-algèbre $\mathbb{B}=\mathbb{P}_{p} \otimes \mathbb{P}_{p+1} \otimes \ldots \otimes \mathbb{P}_{m}$ $(p, m \in \mathbb{Z}, p<m)$ donnée comme un produit tensoriel d'algèbres abéliennes de dimension finie, est associé canoniquement un couplage $\lambda^{\prime}$ de $\mathbb{A}$ avec $\mathbb{X}$, de la manière suivante:

à chaque $k$ est associée une désintégration $\varrho=\sum_{i \in I_{k}} \mu_{i}^{k} \varrho_{i}^{k}$ de $\varrho$ sur l'espace $I_{k}$ des atomes de $\mathbb{P}_{k}$ (cf. 2.1.1), et donc une application $i \rightarrow \varrho_{i}^{k}$ de $I_{k}$ dans $X$; d'où une 
application $\pi$ de l'espace produit des $I_{k}(p \leqq k \leqq m)$ dans le produit $X_{p} \times X_{p+1} \times \ldots \times X_{m}$ de $m-p+1$ copies de l'espace $X$, et donc un morphisme d'algèbres de $\mathbb{B}$ dans la sous algèbre de $\mathbb{X}$ engendrée par les coordonnées comprises entre $p$ et $m$. $\lambda^{\prime}$ est alors définie par simple transport, via ce morphisme (et prolongement arbitraire à $\mathbb{X}$ ).

$\lambda^{\prime}$ a les propriétés suivantes:

1. Pour tout $k$ compris entre $p$ et $m$, la restriction de $\lambda^{\prime}$ à $\mathbb{A} \otimes C(X)$ obtenue via la $k$-ième coordonnée de $X^{\mathbb{Z}}$ est un couplage de type $\Lambda_{v}$ :

2. on a $\left|\varepsilon_{\lambda^{\prime}}\left(\mathbb{A}_{k}, \mathbb{R}_{k}\right)-\varepsilon_{\lambda}\left(\mathbb{A}_{k}, \mathbb{P}_{k}\right)\right| \leqq \varepsilon$, et

$$
\varepsilon_{\lambda^{\prime}}\left(\mathbb{A}_{p}, \ldots, \mathbb{A}_{m} ; \mathbb{R}_{p}, \ldots, \mathbb{R}_{m}\right) \geqq \varepsilon_{\lambda}\left(\mathbb{A}_{p}, \ldots, \mathbb{A}_{m} ; \mathbb{P}_{p}, \ldots, \mathbb{P}_{m}\right)-(m-p+1) \varepsilon
$$

[la quantité $H_{\mu}\left(\bigvee \mathbb{P}_{k}\right)-\sum H_{\mu}\left(\mathbb{P}_{k}\right)$ augmente quand on remplace les $\mathbb{P}_{k}$ par les $\mathbb{R}_{k}$ qui sont plus grossières].

2ème étape. D'après ce qui précède, et d'après la définition de $\kappa, \mathbb{A}_{0}$ et $\varepsilon$ étant fixés, pour tout $n$ asez grand on trouvera un couplage $\lambda_{n}$ de $\mathbb{A}$ avec $\mathbb{X}$ tel que l'on ait

$$
H_{e}\left(\gamma, \theta \circ \gamma, \ldots, \theta^{n} \circ \gamma\right)-\varepsilon_{\lambda_{n}}\left(\gamma, \theta \circ \gamma, \ldots, \theta^{n} \circ \gamma ; \mathbb{R}_{0}, \ldots, \mathbb{R}_{n}\right) \leqq 2(n+1) \varepsilon ;
$$

et donc, pour $n$ assez grand,

$$
\varepsilon_{\lambda_{n}}\left(\gamma, \theta \circ \gamma, \ldots, \theta^{n} \circ \gamma ; \mathbb{R}_{0}, \ldots, \mathbb{R}_{n}\right) \geqq(n+1)(\kappa-4 \varepsilon) .
$$

Pour chaque couple d'entiers $(r, n)$ on considère la quantité

$$
a_{n, r}=\frac{1}{n-r+1} \sum_{k=r}^{n} \varepsilon_{\lambda_{n}}\left(\mathbb{A}_{k}, \bigvee_{j=k-r}^{k} \mathbb{R}_{j}\right),
$$

où $\mathbb{A}_{k}$ désigne le couple $\left(\mathbb{A}_{0}, \theta^{k} \circ \gamma\right)$. Comme les $a_{n, r}$ sont bornés par $\log (d)+\varepsilon$, on peut par un procédé diagonal trouver un sous-ensemble infini $I$ de $\mathbb{N}$ tel que

$$
\lim _{n \rightarrow \infty, n \in I} a_{n, r}=a_{r} \text { existe pour tout } r \in \mathbb{N} .
$$

Les $a_{r}$ tendent en croissant vers une limite $a_{\infty}$ quand $r$ tend vers l'infini, et on fixe $r$ tel que $a_{r} \geqq a_{\infty}-\varepsilon$.

3ème étape. On a donc fixé jusqu'à présent: $\varepsilon, \mathbb{A}_{0}, \gamma, d, \mathbb{R}$ et les partitions $\mathbb{R}_{k}$, les couplages $\lambda_{n}$ de $\mathbb{A}$ avec $\mathbb{X}$, l'ensemble d'indices $I$, et l'entier $r$.

On fixe un entier $p \geqq r$, et $n \in I$, suffisamment grand pour qu'on ait

$$
\varepsilon_{\lambda_{n}}\left(\gamma, \theta \circ \gamma, \ldots, \theta^{n} \circ \gamma ; \mathbb{R}_{0}, \ldots, \mathbb{R}_{n}\right) \geqq(n+1)(\kappa-4 \varepsilon),
$$

soit

$$
H_{\lambda_{n}}\left(\bigvee_{0}^{n} \mathbb{R}_{k}\right)-\sum_{0}^{n} H_{\lambda_{n}}\left(\mathbb{R}_{k} \mid \mathbb{A}_{k}\right) \geqq(n+1)(\kappa-4 \varepsilon)
$$

ou encore

$$
\sum_{0}^{n}\left[H_{\lambda_{n}}\left(\mathbb{R}_{k} \mid \bigvee_{0}^{k-1} \mathbb{R}_{j}\right)-H_{\lambda_{n}}\left(\mathbb{R}_{k} \mid \mathbb{A}_{k}\right)\right] \geqq(n+1)(\kappa-4 \varepsilon),
$$

qui implique

$$
\sum_{0}^{n}\left[H_{\lambda_{n}}\left(\mathbb{R}_{k} \mid \bigvee_{0}^{k-1} \mathbb{R}_{j}\right)-H_{\lambda_{n}}\left(\mathbb{R}_{k} \mid \mathbb{A}_{k} \otimes \bigvee_{0}^{k-1} \mathbb{R}_{j}\right)\right] \geqq(n+1)(\kappa-4 \varepsilon)
$$


soit, pour $n$ assez grand

$$
\sum_{p}^{n}\left[H_{\lambda_{n}}\left(\mathbb{R}_{k} \mid \bigvee_{k-p}^{k-1} \mathbb{R}_{j}\right)-H_{\lambda_{n}}\left(\mathbb{R}_{k} \mid \mathbb{A}_{k} \otimes \bigvee_{k-p}^{k-1} \mathbb{R}_{j}\right)\right] \geqq(n-p+1)(\kappa-5 \varepsilon)
$$

et, d'après le Lemme 2.2:

$$
\sum_{p}^{n}\left[\varepsilon_{\lambda_{n}}\left(\bigvee_{k}^{k} \mathbb{R}_{j}, \mathbb{A}_{k}\right)-\varepsilon_{\lambda_{n}}\left(\bigvee_{k}^{k-1} \mathbb{R}_{j}, \mathbb{A}_{k}\right)\right] \geqq(n-p+1)(\kappa-5 \varepsilon) .
$$

Le travail effectué dans la seconde étape (diagonalisation de la double suite des $a_{n, r}$, et choix de l'entier $r$ ), fournit:

$$
\begin{aligned}
\sum_{p}^{n} \varepsilon_{\lambda_{n}}\left(\bigvee_{k-p}^{k} \mathbb{R}_{j}, \mathbb{A}_{k}\right) & =(n-p+1) a_{n, p} \simeq(n-p+1) a_{p} \simeq(n-p+1) a_{r} \simeq(n-p+1) a_{n, r} \\
& =\frac{n-p+1}{n-r+1} \sum_{r}^{n} \varepsilon_{\lambda_{n}}\left(\bigvee_{k-r}^{k} \mathbb{R}_{j}, \mathbb{A}_{k}\right) \simeq \sum_{p}^{n} \varepsilon_{\lambda_{n}}\left(\bigvee_{k-r}^{k} \mathbb{R}_{j}, \mathbb{A}_{k}\right),
\end{aligned}
$$

où chaque signe $\simeq$ représente une égalité à $(n-p+1) \varepsilon$ près ( $r$ et $p$ sont fixés, $n$ est suffisamment grand dans $I$ ), et donc

$$
\sum_{p}^{n}\left[\varepsilon_{\lambda_{n}}\left(\bigvee_{k-r}^{k} \mathbb{R}_{j}, \mathbb{A}_{k}\right)-\varepsilon_{\lambda_{n}}\left(\bigvee_{k-p}^{k-1} \mathbb{R}_{j}, \mathbb{A}_{k}\right)\right] \geqq(n-p+1)(\kappa-9 \varepsilon)
$$

Pour $k \geqq r$, on pose $\mathbb{P}_{k}=\bigvee_{k}^{k} \mathbb{R}_{j}$, et on applique aux $\mathbb{P}_{k}(r \leqq k \leqq n)$ la procédure établie dans la première étape, pour déduire de chaque $\lambda_{n}$ un nouveau couplage $\lambda_{n}^{\prime}$ de $\mathbb{A}$ avec $\mathbb{X}$, dont chaque restriction à un couple $\left(\mathbb{A}_{k}, \mathbb{R}_{k}\right)$ est de type $\Lambda_{v}$, et pour lequel les quantités $\varepsilon_{\lambda_{n}}\left(\underset{k-1}{\mathbb{A}_{k}}, \mathbb{P}_{k}\right)$ et $\varepsilon_{\lambda_{n}^{\prime}}\left(\mathbb{A}_{k}, \mathbb{R}_{k}\right)$ différent au plus de $\varepsilon$.

Comme la partition $\bigvee_{k-p} \mathbb{R}_{j}$ (considérée dans le couplage $\lambda_{n}$ ), est plus fine que $\bigvee_{k-p+r}^{k-1} \mathbb{R}_{j}$ (considérée dans le couplage $\lambda_{n}^{\prime}$ ), on obtient,

$$
\sum_{p}^{n}\left[\varepsilon_{\lambda_{n}^{\prime}}\left(\mathbb{R}_{k}, \mathbb{A}_{k}\right)-\varepsilon_{\lambda_{n}^{\prime}}\left(\bigvee_{k-p+r}^{k-1} \mathbb{R}_{j}, \mathbb{A}_{k}\right)\right] \geqq(n-p+1)(\kappa-10 \varepsilon)
$$

et, toujours pour $n$ assez grand:

$$
\sum_{p}^{n}\left[\varepsilon_{\lambda_{n}^{\prime}}\left(\mathbb{R}_{k}, \mathbb{A}_{k}\right)-\varepsilon_{\lambda_{n}^{\prime}}\left(\bigvee_{k-p}^{k-1} \mathbb{R}_{j}, \mathbb{A}_{k}\right)\right] \geqq(n-p+1)(\kappa-11 \varepsilon) .
$$

La $C^{*}$-algèbre abélienne $\mathbb{X}$ possède une double dynamique: celle héritée de la dynamique de $\mathbb{A}$, sur chacune de ses composantes $X=S(\mathbb{A})$, et celle induite par le shift sur $X^{\mathbb{Z}}$; on notera $\sigma$ l'automorphisme de $\mathbb{X}$ composé des deux, qui s'écrit sur $X^{\mathbb{Z}}: \sigma\left(\left\{\omega_{n}\right\}_{n \in \mathbb{Z}}\right)=\left\{\theta\left(\omega_{n+1}\right)\right\}_{n \in \mathbb{Z}} ;$ après quoi, en posant $\theta=\theta \otimes \sigma$, la relation ci-dessus se réécrit:

$$
\sum_{p}^{n}\left[\varepsilon_{\lambda_{n}^{\prime} \circ \tilde{\theta}^{k}}\left(\mathbb{R}_{0}, \mathbb{A}_{0}\right)-\varepsilon_{\lambda_{n}^{\prime} \circ \tilde{\theta}^{k}}\left(\bigvee_{-p}^{-1} \mathbb{R}_{j}, \mathbb{A}_{0}\right)\right] \geqq(n-p+1)(\kappa-11 \varepsilon),
$$

et donc, toujours pour $n$ suffisamment grand dans $I$, et $p$ fixé:

$$
\sum_{0}^{n}\left[\varepsilon_{\lambda_{n^{\prime}} \circ \tilde{\theta}^{k}}\left(\mathbb{R}_{0}, \mathbb{A}_{0}\right)-\varepsilon_{\lambda_{n^{\prime}} \circ \tilde{\theta}^{k}}\left(\bigvee_{-p}^{-1} \mathbb{R}_{j}, \mathbb{A}_{0}\right)\right] \geqq(n+1)(\kappa-12 \varepsilon)
$$


On considère alors la mesure moyennée $\lambda_{n}^{\prime \prime}=\sum_{0}^{n} \frac{1}{n+1}\left(\lambda_{n}^{\prime} \circ \ddot{\theta}^{k}\right)$. Si on n'avait moyenné que de $p$ à $n$, les termes $\frac{1}{n-p+1} \sum_{p}^{n} \varepsilon_{\lambda_{n}^{\prime} \cdot \tilde{\theta}^{k}}\left(\mathbb{R}_{0}, \mathbb{A}_{0}\right)$ et $\varepsilon_{\lambda_{n}^{\prime}}\left(\mathbb{R}_{0}, \mathbb{A}_{0}\right)$ ne différeraient que de $\varepsilon$ au plus (d'après 4.1.1); pour $n$ assez grand, les termes $\frac{1}{n+1} \sum_{0}^{n} \varepsilon_{\lambda_{n}^{\prime} \tilde{\theta}^{k}}\left(\mathbb{R}_{0}, \mathbb{A}_{0}\right)$ et $\varepsilon_{\lambda_{n}^{\prime \prime}}\left(\mathbb{R}_{0}, \mathbb{A}_{0}\right)$ différent donc au plus de $2 \varepsilon$.

D'autre part, la moyennisation diminue l'information mutuelle (Lemme 2.3): on a

$$
\frac{1}{n+1} \sum_{0}^{n} \varepsilon_{\lambda_{n}^{\prime} 0 \tilde{\theta}^{k}}\left(\bigvee_{-p}^{-1} \mathbb{R}_{j}, \mathbb{A}_{0}\right) \geqq \varepsilon_{\lambda_{n}^{\prime \prime}}\left(\bigvee_{-p}^{-1} \mathbb{R}_{j}, \mathbb{A}_{0}\right)
$$

d'où

$$
\varepsilon_{\lambda_{n}^{\prime}}\left(\mathbb{R}_{0}, \mathbb{A}_{0}\right)-\varepsilon_{\lambda_{n}^{\prime \prime}}\left(\bigvee_{-p}^{-1} \mathbb{R}_{j}, \mathbb{A}_{0}\right) \geqq \kappa-14 \varepsilon
$$

donc

$$
\varepsilon_{\lambda_{n}^{\prime \prime}}\left(\stackrel{\bigvee}{\bigvee}_{-p}^{0} \mathbb{R}_{j}, \mathbb{A}_{0}\right)-\varepsilon_{\lambda_{n}^{\prime \prime}}\left(\stackrel{\bigvee}{-p}^{-1} \mathbb{R}_{j}, \mathbb{A}_{0}\right) \geqq \kappa-14 \varepsilon ;
$$

ce qui s'écrit encore, par le Lemme 2.2:

$$
H_{\lambda_{n}^{\prime \prime}}\left(\mathbb{R}_{0} \mid \bigvee_{-p}^{-1} \mathbb{R}_{j}\right)-H_{\lambda_{n}^{\prime \prime}}\left(\mathbb{R}_{0} \mid \bigvee_{-p}^{-1} \mathbb{R}_{j} \otimes \mathbb{A}_{0}\right) \geqq \kappa-14 \varepsilon
$$

Cette inégalité est vraie pour tout $p$ fixé, et tout $n$ dans $I$ grand devant $p$. La suite de mesures $\left\{\lambda_{n}^{\prime \prime}, n \in I\right\}$ ne dépend pas de $p$, et elle vérifie $\left\|\lambda_{n}^{\prime \prime}-\lambda_{n}^{\prime \prime} \circ \widetilde{\theta}\right\| \leqq 1 / n$. Comme l'ensemble des états sur $\mathbb{A} \otimes \mathbb{B}$ est compact, tout point d'accumulation de cette suite nous donne un couplage stationnaire $\Lambda$ de $\mathbb{A}$ avec $(\mathbb{B}, \sigma)$ qui vérifie pour tout $p \geqq r$ :

$$
H_{\Lambda}\left(\mathbb{R}_{0} \mid \bigvee_{-p}^{-1} \mathbb{R}_{j}\right)-H_{\Lambda}\left(\mathbb{R}_{0} \mid \mathbb{A}_{0} \otimes \underline{-}_{-p}^{-1} \mathbb{R}_{j}\right) \geqq \kappa-14 \varepsilon,
$$

et donc, à la limite

$$
H_{\Lambda}\left(\mathbb{R}_{0} \mid \mathbb{R}_{0}^{-}\right)-H_{\Lambda}\left(\mathbb{R}_{0} \mid \mathbb{A}_{0} \otimes \mathbb{R}_{0}^{-}\right) \geqq \kappa-14 \varepsilon
$$

soit

$$
h_{\Lambda}\left(\mathbb{R}_{0}, \theta\right)=H_{\Lambda}\left(\mathbb{R}_{0} \mid \mathbb{R}_{0}^{-}\right)-H_{\Lambda}\left(\mathbb{R}_{0} \mid \mathbb{A} \otimes \mathbb{R}_{0}^{-}\right) \geqq \kappa-14 \varepsilon .
$$

La démonstration est terminée.

\section{Spectre singulier implique entropie nulle}

On note $U_{e}$ l'opérateur unitaire dans l'espace de Hilbert $\mathscr{H}_{\varrho}$ caractérisé par la relation $U_{\varrho} \pi_{e}(x) \xi_{\varrho}=\pi_{\varrho}(\theta x) \xi_{\varrho}, \forall x \in \mathbb{A}$.

5.1. Proposition. Si la mesure spectrale de l'opérateur $U_{e}$ est singulière par rapport à la mesure de Lebesgue sur le cercle, l'entropie $h_{\ell}(\theta)$ est nulle. 
Démonstration. $\lambda$ est un couplage stationnaire de $(\mathbb{A}, \theta, \varrho)$ avec $(\mathbb{B}, \sigma)$; on désigne par $\mu$ la restriction de $\lambda$ à $\mathbb{B}$. $\mathbb{P}$ est une partition finie de $\mathbb{B}$. On veut montrer $h(\lambda, \mathbb{P})=0$.

On utilisera la propriété suivante, qui se démontre comme dans le cas classique:

Si la partition $\mathbb{Q}$ est $\bigvee_{-\infty}^{+\infty} \sigma^{i} \mathbb{P}$-mesurable, on a

$$
H\left(\mathbb{Q} \mid \mathbb{A} \otimes \mathbb{Q}^{-}\right) \leqq H\left(\mathbb{P} \mid \mathbb{A} \otimes \mathbb{P}^{-}\right) .
$$

Premier cas. On suppose que le système dynamique $(\mathbb{B}, \sigma, \mu)$ est ergodique. $+\infty$

Par le théorème de Sinaï [5], il existe dans $\mathbb{B}$ une partition finie $\mathbb{Q}$ qui est $\bigvee_{-\infty}^{+\infty} \sigma^{i} \mathbb{P}$-mesurable, et qui engendre un schéma de Bernoulli d'entropie $H\left(\mathbb{P} \mid \mathbb{P}^{-}\right)$. On a $h(\mathbb{Q}, \lambda) \geqq h(\mathbb{P}, \lambda)$ d'après 5.1.1.

On peut supposer maintenant que $\mathbb{B}=\bigvee_{-\infty}^{+\infty} \sigma^{i} \mathbb{Q}$. Soit $\mathscr{H}_{\lambda}$ l'espace de Hilbert de la représentation G.N.S. de $\lambda$, avec vecteur canonique $\Omega_{\lambda}$; soit $U_{\lambda}$ l'unitaire de $\mathscr{H}_{\lambda}$ implémentant canoniquement l'automorphisme $\theta \otimes \sigma: U_{\lambda}(x \otimes b) \Omega_{\lambda}$ $=[\theta(x) \otimes \sigma(b)] \Omega_{\lambda}, \forall x \in \mathbb{A}, b \in \mathbb{B}$.

Pour tout $x$ dans $\mathbb{A}$, le vecteur $x \Omega_{\lambda}$ appartient à la composante singulière du spectre de $U_{\lambda}$; pour tout $b$ dans $\mathbb{B}$, qui est un schéma de Bernoulli, le vecteur $[b-\mu(b))] \Omega_{\lambda}$ appartient à la composante "Lebesgue» de ce même unitaire: les deux vecteurs sont donc orthogonaux; ce qui donne

$$
\lambda(x \otimes b)-\varrho(x) \mu(b))=\left\langle[x \otimes(b-\mu(b))] \Omega_{\lambda}, \Omega_{\lambda}\right\rangle=\left\langle[b-\mu(b)] \Omega_{\lambda}, x^{*} \Omega_{\lambda}\right\rangle=0 ;
$$

soit $\lambda=\varrho \otimes \mu$, et $h(\mathbb{Q}, \lambda)=0$; donc a fortiori $h(\mathbb{P}, \lambda)=0$.

Cas général. Considérons l'algèbre $L^{\infty}(\mathbb{B}, \mu)^{\sigma}$ des fonctions mesurables bornées $\sigma$-invariantes sur le spectre de $\mathbb{B}$, et écrivons-la $L^{\infty}(Z, v)$ où $v$ est une mesure de probabilité sur l'espace compact $Z$.

On obtient une désintégration $\lambda=\int_{Z} \lambda_{z} d v(z)$ de $\lambda$ en états $\theta \otimes \sigma$ invariants sur $\mathbb{A} \otimes \mathbb{B}$, chaque $\lambda_{z}$ fournissant un couplage entre un système dynamique $\left(\mathbb{A}, \theta, \varrho_{z}\right)$ et un système dynamique classique ergodique $\left(\mathbb{B}, \sigma, \mu_{z}\right)$. On remarquera que chacun des $\left(\mathbb{A}, \theta, \varrho_{Z}\right)$ hérite de la propriété de spectre singulier.

On considère le système dynamique composé de la $C^{*}$-algèbre $C(Z) \otimes \mathbb{A}$, de l'automorphisme $\tilde{\theta}=$ identité $\otimes \theta$, et de l'état $\tilde{\varrho}=\int_{Z}\left(\delta_{z} \otimes \varrho_{z}\right) d v(z)$; puis le couplage $\tilde{\lambda}=\int_{Z}\left(\delta_{z} \otimes \lambda_{z}\right) d v(z)$ avec $(\mathbb{B}, \sigma)$.

On a d'abord $H_{\tilde{\lambda}}\left(\mathbb{P} \mid C(Z) \otimes \mathbb{A} \otimes \mathbb{P}^{-}\right)=\int_{Z} H_{\lambda_{z}}\left(\mathbb{P} \mid \mathbb{A} \otimes \mathbb{P}^{-}\right) d v(z)$ et donc $h(\tilde{\lambda}, \mathbb{P})$ $=\int_{Z} h\left(\lambda_{z}, \mathbb{P}\right) d v(z)=0$ (d'après le cas ergodique ci-dessus).

On a ensuite $H_{\tilde{\lambda}}\left(\mathbb{P} \mid C(Z) \otimes \mathbb{A} \otimes \mathbb{P}^{-}\right)=\lim _{m \rightarrow \infty} H_{\tilde{\lambda}}\left(\mathbb{P} \mid \mathbb{C}_{m} \otimes \mathbb{A} \otimes \mathbb{P}^{-}\right)$, où les $\mathbb{C}_{m}$ sont des algèbres abéliennes de dimension finie sur lesquelles $\tilde{\theta}$ agit trivialement (parce que $C(Z)$ est nucléaire, et d'après $[3$, lemme I.1, (5)]). Puis, pour chaque $m$,

$$
H_{\tilde{\lambda}}\left(\mathbb{P} \mid \mathbb{C}_{m} \otimes \mathbb{A} \otimes \mathbb{P}^{-}\right)=\lim _{n \rightarrow \infty} \frac{1}{n+1} H_{\tilde{\lambda}}\left(\bigvee_{0}^{n} \sigma^{i} \mathbb{P} \mid \mathbb{C}_{m} \otimes \mathbb{A}\right)=H_{\lambda}\left(\mathbb{P} \mid \mathbb{A} \otimes \mathbb{P}^{-}\right)
$$

en appliquant le Lemme 2.2 pour la seconde égalité.

D'où finalement $h(\lambda, \mathbb{P})=h(\widetilde{\lambda}, \mathbb{P})=0$. (c.q.f.d.) 


\section{Bibliographie}

1. Araki, H.: Relative entropy for states of von Neumann algebras. Publ. RIMS Kyoto 11, 809-833 (1976)

2. Connes, A.: Entropie de Kolmogorov-Sinaï et mécanique statistique quantique. Comptes Rendus Acad. Sci. Paris, I 301, 1-6 (1985)

3. Connes, A., Narnhofer, H., Thirring, W.: Dynamical entropy of $C^{*}$-algebras and von Neumann algebras. Commun. Math. Phys. 112, 691-719 (1987)

4. Connes, A., Størmer, E.: Entropy of automorphisms of $I I_{1}$ von Neumann algebras. Acta Math. 134, 289-306 (1975)

5. Sinaï, J.G.: Weak isomorphism of transformations with invariant measure. Math. Sbornik 63/105, 23-42 (1964)

6. Størmer, E., Voiculescu, D.: Entropy of Bogoliubov automorphisms of the canonical anticommutation relations. Commun. Math. Phys. 133, 521-542 (1990)

7. Voiculescu, D.: Entropy of dynamical systems and perturbation of operators (à paraître)

8. Ohya, M.: Some aspects of quantum information theory. Rep. Math. Phys. 27, 19-47 (1989)

Communiqué par A. Connes 
